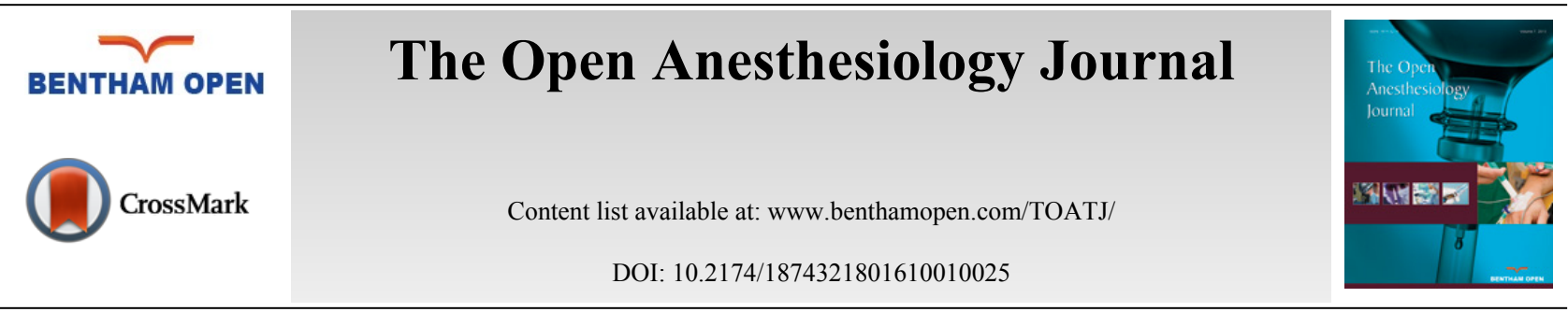

BOOK REVIEW

\title{
Book Review: Drugs in Anaesthesia and Intensive Care
}

\author{
Rakesh Garg,\# \\ Intensive Care, Pain and Palliative Care, All India Institute of Medical Sciences, New Delhi-110029, India
}

The fifth edition of the book "Drugs in Anaesthesia and Intensive Care" has been thoroughly revised with addition of newer drugs used in perioperative and critical care management. The book comprehensively summarized all the relevant drugs required in perioperative period and critical care settings.

The description of drugs has been paragraphed and bulleted for all relevant properties of the drug required for its understanding. This makes it useful not only for acquiring knowledge easily, but also the subheading makes it prompt reference for the specific use in time of need of the drug usage in clinical practice. The search within the text book has been made easier. The drugs are indexed in two ways: based on name of the drug and also based on uses of the drugs. This allows easy search of an individual drug and also easy search of all drugs for a specific use. This allows the user to refer with ease and promptness in times of need. The editors (authors) have very concisely but comprehensively documented all relevant details about the drug. It would help clinicians, paramedics including nursing personnel to use these drugs with better understanding. It would help them for the cautions in specific groups of person due to associated co morbidity. It would allow them to look for any adverse effects. This goes by well known dictum "Eyes sees what the brain knows". Hence identifying drug related issues would be better managed. Also descriptions of the systemic effects of drugs have been briefly described. This would allow observing for the systemic effects specifically and further managed if body functions become compromised. This would also prepare the clinicians and nursing staff to make the adequate arrangements based on the systemic effects of the drugs prior to its administration. The pharmacokinetics would help to cautiously use the drugs in patients having systemic disease affecting liver metabolism or kidney excretion of the drugs. The dosage may require alteration by better drug pharmacokinetics understanding. At times, preparation of drugs for infusion and its administration may lead to mishaps. This hand book has described the drugs precisely and evidence based for various aspects of drugs including method for drug dilution, its administration including its speed and duration for infusion. This is of utmost important specifically with highly potent drug or drugs with significant adverse drug profile and may cause harm if not administered as per norms. The section on 'special points' appears to be a very important clinical pearl. No drugs are without special precautions for its use. It may relate to its use, dosages, routes, associated patient co-morbidity or drug interaction. The editors have concisely included all these clinical pearls and key points comprehensively under this heading.

The authors have provided comparative tables of various drugs for a class. It is ready referral and easy to compare the drugs. However, it would have been good if further tables for various drugs classification may also be added.

This hand book would be of utmost use not only for perioperative physicians, anesthesiologists, Intensivist but also for surgeons and nursing staffs. This would be handy book for all trainees not only for their clinical practice but also for examination preparation. This book would not be a replacement for standard textbook for those requiring in depth knowledge and for trainees (who ought to have detailed knowledge and understanding). However, this book would

\footnotetext{
* Address correspondence to this author at the Intensive Care, Pain and Palliative Care, All India Institute of Medical Sciences, New Delhi-110029, India; Tel: +91-9810394950,+919868398335; E-mail: drrgarg@hotmail.com

\# Associate Editor: Journal of Anaesthesiology Clinical Pharmacology, Indian Journal of Anaesthesia, Journal of Medical Case Reports
} 
indeed provide them a ready and fast revision when required for the purpose of examination for revision or for patient management in acute clinical settings. The style and size for the handbook is truly fit for its urgent use.

Overall, the book is well written, user friendly, easy to carry and refer, updated and comprehensive with regards to drugs required in perioperative period and critical care settings. This book would be important academic armamentarium for anesthesiologist, Intensivist, surgeons, emergency physicians, nurses and paramedics.

(C) Rakesh Garg; Licensee Bentham Open

This is an open access article licensed under the terms of the Creative Commons Attribution-Non-Commercial 4.0 International Public License (CC BY-NC 4.0) (https://creativecommons.org/licenses/by-nc/4.0/legalcode), which permits unrestricted, non-commercial use, distribution and reproduction in any medium, provided the work is properly cited. 